\title{
Estado del conocimiento de los ecosistemas de las sabanas inundables en la Orinoquia Colombiana
}

\author{
State of the knowledge of the flooded savanna ecosystems \\ of Orinoquia Colombiana
}

\section{Estado do conhecimento sobre os ecossistemas das savanas inundadas no Orinoquia Colombiana}

\author{
Carolina Mora-Fernández ${ }^{1}$, Lourdes Peñuela-Recio ${ }^{2}$, Francisco Castro-Lima ${ }^{3}$
}

1 Biologa, Universidad Nacional. La Palmita Centro de Investigación. Grupo de Investigaciones Territoriales para el usos y conservación de la Biodiversidad.

2 Zoot, MSc Desarrollo Sostenible de Sistemas Agrarios, Universidad Javeriana. Fundación Horizonte Verde, Grupo de Investigación Sistemas Sostenibles de Producción con énfasis en Palmas Tropicales - Universidad de Los Llanos.

3 Ing Agrónomo, Universidad de los Llanos. Fundación Horizonte Verde. Grupo de Investigación Sistemas Sostenibles de Producción con énfasis en Palmas Tropicales - Universidad de Los Llanos.

Email: investigacion@lapalmita.com.co

Recibido: mayo 02 de 2013

Aceptado: julio 29 de 2015

\section{Resumen}

Las sabanas inundables corresponden al $12.5 \%$ del área de la cuenca del Orinoco, siendo este un ecosistema estratégico de gran importancia económica, biológica y ecológica. El objetivo de nuestro artículo fue actualizar e informar al lector sobre el estado actual del conocimiento de los ecosistemas de sabanas inundables presentes en la Orinoquia colombiana. Tratamos las siguientes categorías temáticas: estudios de ecosistemas, flora y fauna; conservación y uso sostenible; herramientas de gestión y planeación; y educación ambiental. Analizamos la información teniendo en cuenta el tipo de publicación: artículo, libro, capítulo delibro, informe técnico, tesis y resúmenes. Incluimos un total de 159 publicaciones. El 53\% del número de publicaciones corresponde a literatura publicada oficialmente y el restante $47 \%$ a literatura gris. El mayor porcentaje de las publicaciones corresponde a estudios de ecología, flora y fauna (68\%). En general, consideramos que hacen falta estudios mejor estructurados, que incluyan los ecosistemas de sabanas y su vegetación asociada, ya que la mayoría de los estudios están enfocados a los bosques de galería. Observamos en el análisis por décadas que la mayor contribución al conocimiento de las sabanas inundables de la Orinoquia colombiana fue en las décadas de 1990-2000 y 2000-2010. Este estudio nos confirma la hipótesis que teníamos sobre la poca información disponible que existe para el paisaje de sabana inundable. Algunos grupos taxonómicos han sido mejor estudiados que otros. La estacionalidad es un factor determinante en la dinámica hidrológica de las sabanas inundables. Por esto, consideramos fundamental empezar a trabajar sobre el servicio de regulación hídrica y su importancia en la dinámica y procesos ecológicos, y además profundizar en el conocimiento, conservación y uso de la sabana inundable de la Orinoquia colombiana, porque es un ecosistema estratégico, para la dinámica hidrológica del tercer sistema ribereño más importante del mundo: La cuenca del Orinoco.

Palabras clave: ecosistemas, flora, fauna, Orinoco, regulación hídrica.

\begin{abstract}
Flooded savannas are $12.5 \%$ of the Orinoco basin, a strategic ecosystem of great economical, biological, and ecologic importance. The aim of our study was to update and inform the reader on the state of knowledge of the ecosystems associated to flooded savannas of the Colombian Orinoquia. We worked on the following theme categories: ecosystem
\end{abstract}


studies, flora and fauna, sustainable conservation and use, planning and management tools, and environmental education. We analyzed information according to publication type: scientific paper, book, book chapter, technical report, theses, and summaries. We included a total of 159 publications. 53\% of the literature has been officially published, while the remaining $47 \%$ is still unpublished. The largest amount of publications corresponds to ecological studies, and flora and fauna (68\%). In general, we consider that better structured studies are lacking, ones that include savanna ecosystems, and their associated vegetation, since most of the studies have centered on riparian forests. The decades of 1990-2000 and 2000-2010 harvested the largest amounts of literature. We confirmed our previous hypothesis about the lack of information on flooded savanna ecosystems. Some taxonomic groups have been more thoroughly studied than others. Seasonality is a determinant factor on the hydrologic dynamic of these savannas. Thus, we consider imperative to start working on hydrologic regulation services, and their importance on the dynamic and ecological processes. It is indispensable to further improve the knowledge, use and conservation of the flooded savannas of the Colombian Orinoquia, because this ecosystem is strategic for the hydrologic dynamic of the third most important river system in the World: the Orinoco basin.

Key Words: ecosystems, flora, fauna, Orinoco, hydrologic dynamic.

\section{Resumo}

As savanas inundadas correspondem a 12,5\% da área da bacia do Orinoco, sendo este um ecossistema estratégico de grande importância econômica, biológica e ecológica. O objetivo do nossotra balhofo i atualizar e informar o leitor sobre o estado atual do conhecimento sobre os ecosistemas das savanas inundadas presentes no Orinoco colombiano. Cobrimos as seguintes categorias temáticas: estudos de ecossistemas, flora e fauna; conservação e uso sustentável, ferramentas de gestão e planejamento; e educação ambiental. Analisamos os dados tendo em conta o tipo de publicação: artigos, livros, capítulos de livros, relatórios técnicos, teses e resumos. Incluímos um total de 159 publicações. O 53\% do número de publicações corresponde a literatura publicada oficialmente e os restantes $47 \%$ com a literatura cinza. O maior percentual de publicações corresponde a estudos de ecologia, flora e fauna (68\%). Emgeral, consideramos que são necessários estudos melhores truturados, que contenham ecossistemas de savana e seu vegetação associada, já que a maioria dos estudos se concentram nas matas de galería. Observamos na análise por décadas que a maior contribuição para o conhecimento das savanas inindadas do Orinoquia colombiana foi nas décadas de 1990-2000 e 2000-2010. Este estudo confirma a hipótese que tinha mos sobre a pouca informação disponível que existe para a paisagem de savana inundada. Alguns grupos taxonómicos tem sido melhor estudados do que outros. A sazonalidade é umfator determinante na dinâmica hidrológica das savanas inundadas. Para isso, é fundamental para começar a trabalhar no serviço de regulação da água e sua importância na dinâmica e processos ecológicos. Para este, consideramos fundamental começar a trabalhar no serviço de regulação hídrica e sua importância na dinâmica e os processos ecológicos. Aprofundar no conhecimiento, conservação e uso da savana inundada da Orinoquia colombiana, porque é um ecossistema estratégico para a dinâmica hidrológica do terceiro sistema fluvial mais importante do mundo: A bacia do Orinoco.

Palavras Chave: ecossistemas, flora, fauna, Orinoco, regulação hídrica.

\section{Introducción}

Las sabanas naturales constituyen uno de los biomas más característicos del cinturón intertropical del planeta. Es una de las grandes unidades estructurales y funcionales en las que se ha diferenciado la biota de la tierra, al mismo nivel que las selvas, los bosques montanos, los páramos o los desiertos (Rippstein et al., 2001).

Las sabanas representan el $43 \%$ de la superficie terrestre, ocupando grandes regiones de Suramérica, África, Sureste Asiático y Australia. Se caracterizan por ser asociaciones de vegetación herbácea con presencia, o no, de árboles esparcidos y con patrones estaciónales de disponibilidad de agua determinados por una marcada estación climática seca (Hernández et al., 2005, Mora-Fernández et al., 2011). Las sabanas en Suramérica se extienden con una superficie de 269 millones de hectáreas de las cuales 204 millones las encontramos representadas en el Cerrado de Brasil, 23 millones en los Llanos Orientales Colombianos, 25 millones en los
Llanos de Venezuela, 4 millones en las sabanas de Guyana y 13 millones en las sabanas de Bolivia (Rippstein et al., 2001).

Los Llanos Orientales de Colombia u Orinoquia colombiana contiene tres sistemas de tierras o paisajes: El piedemonte, las llanuras aluviales y las altillanuras. Pertenecen a un macrosistema de importancia global (las sabanas tropicales) cuya topografía permite la mecanización agrícola y sus suelos exigen un manejo cuidadoso (Rippstein et al., 2001). No toda esa superficie es apta para ser cultivada; en buena extensión los suelos presentan limitaciones físicas y químicas, que junto con la marcada estacionalidad climática reducen el espectro de posibilidades para la producción agrícola (Hernández et al., 2005, Mora-Fernández et al., 2011).

Las sabanas inundables de la Orinoquia Colombina cubren la mayor extensión de los departamentos de Arauca y Casanare, hacen parte de la región de los llanos Orientales la cual se caracteriza por la dominancia 
de las sabanas tropicales, formaciones abiertas, con un dosel discontinuo donde predomina una matriz herbácea interrumpida compuesta por pastos y hierbas, o en ocasiones con plantas leñosas de bajo porte y palmas dispersas. (Sarmiento, 1994). En menor extensión se presentan bosques asociados a las riberas y vegas de los ríos, donde la escasez hídrica es alta en la época de sequía, sin embargo, proporciona los niveles mínimos para el mantenimiento de las coberturas vegetales. Estos tipos fisonómicos de vegetación presentan una gran variedad de comunidades vegetales, asociadas a cambios en topografía y tipo de suelo en la región.

Las sabanas inundables corresponden al $12.5 \%$ del área de la cuenca del Orinoco, siendo este un ecosistema estratégico de gran importancia económica, biológica y ecológica para toda la Orinoquia. Este tipo de sabanas se caracterizan por establecerse en un plano topográfico cóncavo con zonas muy bajas conocidas como bajos y esteros, los cuales en épocas de lluvias se inundan por acción directa de la precipitación y por la escorrentía dada por el desborde de los ríos y caños. Por otro lado, las áreas más altas, se conocen como bancos y banquetas que constituyen la base forrajera para la ganadería y fauna silvestre (Sastre et al., 2006).

Nuestro artículo tiene como objetivo actualizar e informar al lector sobre el estado actual del conocimiento de los ecosistemas de sabanas inundables presentes en la Orinoquia Colombiana. Presentamos integralmente las principales características de las sabanas inundables tomando en cuenta aspectos como: ubicación geográfica, biodiversidad, factores de cambio y uso del territorio. Finalmente, analizamos las fuentes de información disponibles a la fecha, así como la información reportada por estas; los vacíos existentes y los temas que deben ser solventados en estudios futuros.

\section{Materiales y métodos}

\section{Ámbito del estudio}

Estudiamos el estado del conocimiento de los ecosistemas de las sabanas inundables de la Orinoquia Colombiana, para los departamentos de Arauca y Casanare, en las siguientes categorías temáticas:

- Estudios de ecosistemas, flora y fauna

- Conservación y uso sostenible

- Herramientas de gestión y planeación

- Educación ambiental

\section{Obtención de datos}

Realizamos la búsqueda a través de google ${ }^{\circledR}$, en su módulo académico, en bases de datos bibliográficas de la Universidad Nacional de Colombia, Pontificia Universidad Javeriana, Universidad de los Andes, Universidad de los Llanos, Universidad para el Trópico Colombiano (UNITROPICO), Instituto Alexander von Humboldt (IAvH), Centro de documentación del Instituto de Hidrología, Meteorología y Estudios Ambientales (IDEAM, así como las bases de datos en línea de los centros de documentación de las Corporaciones Autónomas Regionales (CARs) utilizando palabras claves como: sabanas inundables, sabanas tropicales, llanos orientales, Colombia, macrófitas, flora acuática, peces, aves, mamíferos, anfibios, reptiles, procesos productivos, conservación y su combinación.

En la búsqueda incluimos documentos de inventarios de fauna y flora, temas relacionado con grupos focales (aves, mamíferos, reptiles, anfibios, peces) y plantas vasculares. Lo anterior debido a que son grupos que generalmente se tienen en cuenta para realizar evaluaciones rápidas de cualquier sistema ecológico y que por lo tanto son criterios a la hora de la toma de decisiones sobre el uso de estos ecosistemas para diversos fines, como son: la conservación, proyectos de infraestructura o aprovechamiento de recursos naturales no renovables (minería y petrolero).

Así mismo, consultamos documentos sobre aspectos ecológicos, de manejo, conservación y sistemas productivos por ser acercamientos complementarios a los anteriores que manejan otra escala de análisis importante para el estudio.

\section{Análisis de la información}

Analizamos la información teniendo en cuenta el tipo de publicación: 1) Artículo: son documentos cortos, divulgados en una publicación seriada debidamente reconocida a través del Número Internacional Normalizado de Publicaciones Seriadas (ISSN por sus siglas en ingles). 2) Libro: son documentos extensos identificados con un Numero Estándar Internacional de Libro (ISBN por sus siglas en ingles). 3) Capítulo de libro: son contribución hecha en el marco de un libro. 4) Informe técnico: es una contribución realizada en el marco de un contrato de consultoría y que no se publicó formalmente como artículo o libro. 5) Tesis, es un trabajo en extenso, producto de una investigación para optar por un título profesional de pregrado o posgrado y que no se publicó formalmente como libro o artículo. 6) Resumen, son resúmenes de trabajos presentados en 
eventos como congresos que hacen parte de memorias, simposios, conferencias, entre otros.

De igual forma consideramos las siguientes categorías temáticas: 1) Estudios de ecosistemas, flora y fauna, incluye inventarios, estudios relacionadas con la ecología y dinámicaen ecosistemas de sabanas inundables en los departamentos de Arauca y Casanare; estudios que caractericen la flora; estudios en aves, mamíferos, anfibios, reptiles y peces que incluían todos los estudios que evaluaban exclusivamente a cada uno de estos grupos faunísticos, sus especies, atributos ecológicos, distribución y aprovechamiento. 2) Conservación y uso sostenible, en esta temática se incluyeron todos los estudios que realizaron diagnósticos ambientales, procesos de zonificación, descripción de problemáticas, amenazas y consideraciones generales para el manejo de los sectores, productivos y económicos. 3) Herramientas de gestión y planeación,en esta temática incluye todos los estudios que realicen planes de manejo ambiental, descripción de problemáticas, amenazas, planes de ordenamiento de cuencas, políticas ambientales para la Orinoquia, entre otros. 4) Educación ambiental, incluye temáticas relacionadas con educación ambiental en torno a las sabanas inundables.

\section{Las sabanas inundables de la orinoqua colombiana}

\section{Contexto geográfico:}

\section{Localización}

La región de los llanos orientales colombianos, conocida como Orinoquía, comprende la parte norte de las Ilanuras orientales de Colombia, cuenca occidental del río Orinoco, región plana de baja altitud, correspondiente al 30,4\% del territorio nacional (Romero et al., 2004; Romero-Ruiz et al., 2012). Sus sabanas se caracterizan por el clima intertropical. La región limita al norte y al oriente con la Republica de Venezuela, al sur con la región amazónica de Colombia y al occidente con la cordillera Oriental (cordillera de los Andes). Comprende los departamentos de Arauca, Casanare, Meta, Vichada y Norte del Guaviare (IGAC, 2008).

Los llanos de Colombia presentan una gran diversidad de paisajes, que van desde las zonas de piedemonte y terrazas en los márgenes de la Cordillera Oriental hasta las llanuras que se extienden por el oriente. De forma general, Goosen $(1964,1971)$ describe cinco tipos de paisajes: 1) el piedemonte, 2) las terrazas, 3) la llanura aluvial de desborde, 4) la llanura eólica, 4) la altillanura y 5) los aluviones recientes como se aprecia en la Figura 1.

Las sabanas inundables se ubican en los departamentos de Arauca y Casanare al oriente del territorio colombiano en la parte del flanco nororiental de la cordillera oriental y limite occidental de la región de la Orinoquía. Los paisajes que cubren la mayor extensión de estos dos departamentos son las llanuras, que en conjunto conforman la planicie aluvial o llamada regionalmente los Llanos de Arauca - Casanare (IGAC, 1993). La planicie aluvial es una región donde los ríos que vienen de la cordillera han perdido su capacidad de carga y sólo llevan en suspensión sedimentos finos; en consecuencia los cauces que venían encajonados y profundos en la cordillera y el piedemonte se transforman en lechos amplios y de poca profundidad, lo cual favorece la ocurrencia de fenómenos como desbordamientos, inundaciones y cambios de curso, especialmente durante la época de lluvias, en la que es importante evacuar toda el agua que se almacena en la llanuras (IGAC, 2008).

\section{Tipos de Sabanas inundables en Arauca y Casanare}

La historia geológica de los Llanos de Colombia se encuentra directamente asociada a los levantamientos y hundimientos de la Cordillera Oriental (Goosen, 1964; Botero, 1999); siendo los últimos levantamientos del Terciario y del Cuaternario los más interesantes, porque estos dieron lugar a los grandes procesos de erosión en la Cordillera, siendo causa y fuente de la sedimentación en los Llanos (Mora-Fernández et al., 2011).

Las sabanas inundables son clasificadas como sabanas hiperestacionales, en donde sus condiciones climáticas y de estrés hídrico le confieren características particulares (Sarmiento 1984; Romero et al., 2004). Ramia en 1967 las define según el tipo de inundación en la época de lluvias en bancos o sitios altos no inundables, bajos o sitios que se inundan y esteros que anegan profundamente.

Huber y Alarcón (1988) agrupan las sabanas tropicales en dos tipos: las sabanas abiertas inundables y las sabanas abiertas estacionalmente inundables. La primera se caracteriza por presentar un estrato herbáceo ralo y bajo, dominado por gramínea densas inundable durante la mayor parte del año con presencia de palmares leñosos, y la segunda por la ausencia de elementos leñosos.

En un estudio más reciente realizado por el Instituto de Estudios Ambientales de la Universidad Nacional 


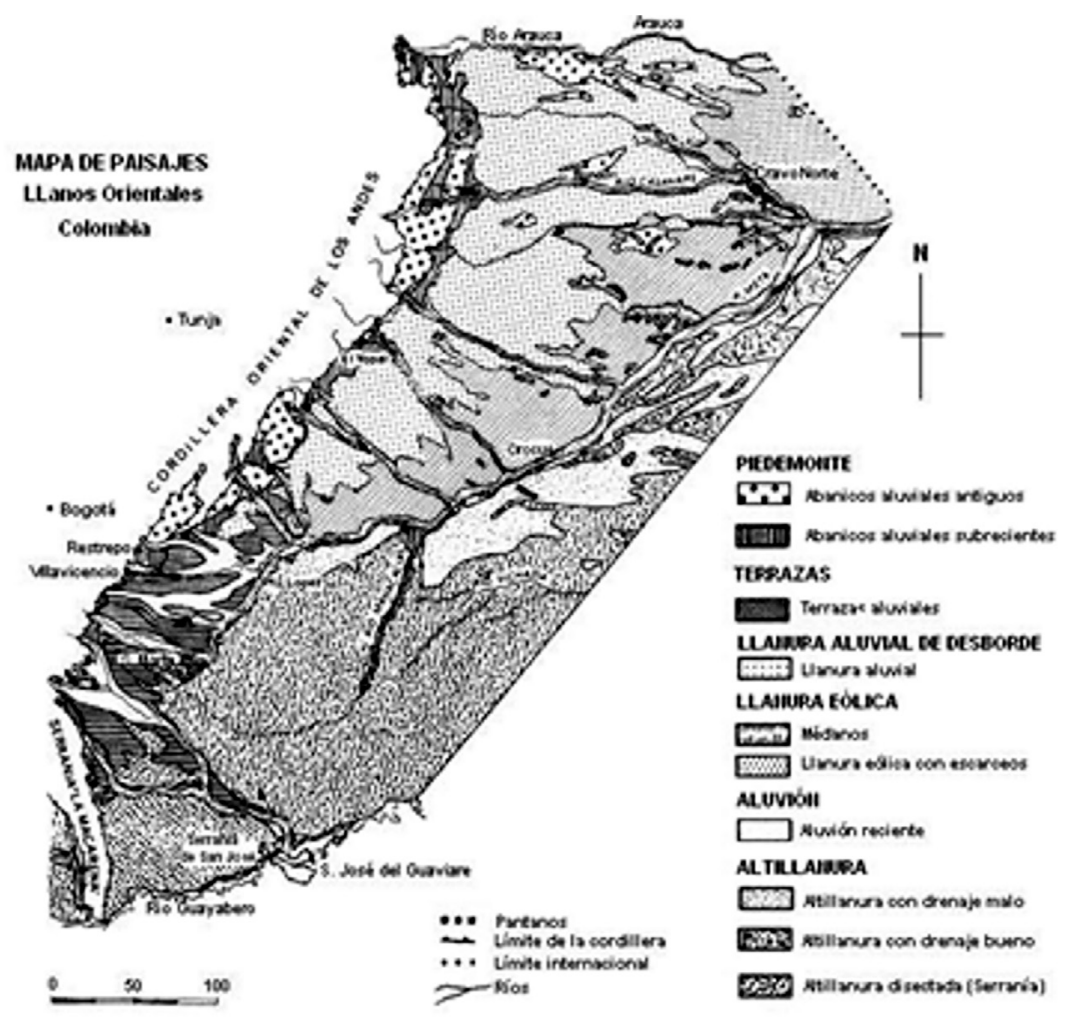

Figura 1. Mapa de paisajes. Adaptado de Goosen, 1964, 1971.

(2011) en las sabanas inundables asociadas a la cuenca baja del rió Pauto, dividen el paisaje en dos grupos amplios de acuerdo a su ubicación relativa con el río Pauto: las sabanas ubicadas en la vega del río Pauto y la llanura aluvial de desborde, y las sabanas ubicadas en la llanura eólica. Se reportan variaciones en la vegetación asociada a los rasgos geomorfológicos, a los tipos de suelo y al de uso que le dan los habitantes de la región (Pinzón-Pérez, et al., 2011 y Mora-Borrás 2011)

\section{Dinámica hidrológica de las sabanas inundables}

La estacionalidad hidrológica es la fuerza motriz que provoca los cambios en los patrones y procesos ecológicos que ocurren en los ecosistemas inundables de los grandes ríos tropicales. Los regímenes hidrológicos naturales de los ríos modulan el funcionamiento de estos ecosistemas de muchas maneras, incluyendo el modelamiento geomorfológico del paisaje fluvial y la provisión de señales ambientales que rigen los ciclos de la vida de los seres vivos (Montoya et al., 2011).

Los grandes ríos de las planicies inundables tropicales son sistemas muy complejos con alta productividad biológica y elevada heterogeneidad espacio temporal en estructura y procesos a múltiples escalas (Welco- me, 1979; Junk et al, 1989; Neiff 1990; Tockner y Stanford, 2002; Montoya et al., 2011).

La importancia del mantenimiento de la dinámica hidrológica natural de los grandes ríos con planicie inundable no fue entendida claramente hasta la aparición de los modelos e hipótesis que describían explícitamente el funcionamiento de estos ecosistemas (Junk et al., 1989; Poff et al., 1997; Junk y Watzen, 2004), por lo que la degradación de éstos debido a su explotación no era considerada como una actividad negativa. Por el contrario, el aprovechamiento de estos ecosistemas a través de la modificación de sus regímenes hidrológicos naturales mediante obras de ingeniería esta visto como un logro importante y de mucho valor dentro de la visión desarrollista de las sociedades (Novo, 2002; Postel y Richter, 2003).

El régimen hidrológico de los grandes ríos tropicales presentan una alta estacionalidad mediada por un patrón temporal de las precipitaciones, el cual está determinado principalmente por el movimiento latitudinal de la zona de convergencia intertropical a lo largo del año (Winemiller, 2004; William M, 2008). La mayoría de los grandes ríos tropicales exhiben un régimen hidrológico unimodal anual, con marcadas diferencias entre los periodos secos y los de lluvias, causando la 
presencia de un único pulso de inundación. Aunque el pulso de inundación es bastante predecible en los grandes ríos tropicales (Schöngart y Junk, 2007), el fenómeno del Niño afecta los patrones de precipitación en las zonas tropicales, produciendo variaciones interanuales en los patrones de caudal (Foley et al., 2002; Molinier et al., 2009; Montoya et al., 2011).

Estos pulsos de inundación son la fuerza motriz que modula los cambios anuales de las variables bióticas y abióticas que tienen lugar en el canal principal y en todos los cuerpos de agua asociados a la planicie inundable. Una alta complejidad geomorfológica de esto sistemas (lo que origina a su vez una alta heterogeneidad de hábitats), una elevada productividad biológica y una importante biodiversidad son mantenidas en el tiempo gracias a la acción de las inundaciones periódicas (Montoya et al., 2011). Estas ideas fueron desarrolladas y ensambladas en lo que se conoce como el concepto del "Pulso de Inundación" (Junk et al., 1989; Junk y Wantzen, 2004). El pulso es generado por la inundación periódica, seguida de una fase de sequía. Este concepto define la planicie de inundación como la zona de transición acuático-terrestre, ya que ésta alterna entre estos dos tipos de ambientes. Por tanto los ríos y sus planicies de inundación no deben ser analizadas separadamente (Junk et al., 1989; Junk y Wantzen, 2004). Este concepto señala la importancia de la conectividad hidrológica lateral entre el canal principal y la gran variedad de cuerpos de agua existentes, en donde el intercambio de materia orgánica entre la planicie de inundación, lagunas, caños y ríos durante los pulsos de inundación contribuye en gran medida a sustentar la alta productividad biológica de las zonas inundables. Esta conectividad lateral, la cual cambia estacionalmente, origina una variedad espacio-temporal importante reflejada por la presencia de parches o hábitats que se interrelacionan en grados variables siguiendo el concepto de "Mosaico de hábitats cambiantes" (SHM, de shifting hábitat mosaic) de Stanford et al., (2005).

Los regímenes hidrológicos son responsables de la renovación de hábitats, de sustentar la variabilidad estacional en los procesos de productividad primaria y de ciclaje de nutrientes, de proveer y asegurar las señales ambientales que son necearías para el éxito reproductivo y de reclutamiento (persistencia en el tiempo) de los organismos y del mantenimiento de la variabilidad espacio-temporal entre el cuerpo de agua en el ecosistema (Montoya et al., 2011).

Ahora bien, la ocupación humana de las planicies inundables tropicales se han debido a la gran provisión de bienes y servicios que estos ecosistemas ofrecen (Mon- toya et al., 2011), sin embargo su aprovechamiento ha estado asociada al control de los cambio ambientales cíclicos originados por la estacionalidad hídrica propia de los estos ecosistemas. La explotación intensiva de los recursos y servicios de estos ecosistemas han estado estrechamente vinculado con la alteración de la dinámica hidrológica de los ríos, originando en la mayoría de los casos la disminución de su potencial aprovechable colocándolo entre los ecosistemas más amenazados del mundo (Allan yFlecker, 1993; Bayley, 1995; Tockner y Stanford, 2002; Postel y Richer, 2003)

Las alteraciones antrópicas a estos regímenes hidrológicos naturales afectan tanto la capacidad de sustentar los patrones y procesos ecológicos así como de satisfacer las necesidades humanas en estos ecosistemas. Finalmente, estas modificaciones responden a la necesidad de controlar las inundaciones en la mayoría de los casos. Sin embargo, no siempre las sociedades obtienen los resultados esperados y se crean problemas ambientales mayores por modificar el régimen hidrológico de los ríos (Montoya et al., 2011).

Por lo tanto, es necesario tener un enfoque integral de manejo para estos ecosistemas, con el fin de no controlar la naturaleza para explotar todo su potencial. Deben diseñarse planes y estrategias, con los diversos actores, que nos permitan aprovechar los recursos y el mantenimiento de los servicios ecosistemicos a través del tiempo.

\section{Estudios de ecosistemas, flora y fauna en las sabanas inundables en la orinoquia colombiana}

En la Orinoquía, la diversidad biológica se explica a través de la convergencia de varios fenómenos evolutivos y la gran movilidad espacio-temporal de los límites biogeográficos de las selvas y sabanas. Eventos glaciares e interglaciares modificaron la distribución de estos ecosistemas, los cuales funcionaron como rutas, corredores y áreas de dispersión de especies provenientes de las selvas circundantes. Distintos elementos bióticos confluyeron en grandes áreas que han funcionado como ecotonos (zonas de transición entre Orinoquía-Amazonía, Orinoquía-Andes) (Correa et al., 2006). Los Llanos Orientales representan, por lo tanto, una mezcla de fauna y flora proveniente de varios biomas adyacentes (Ojasti 1990, Defler y Rodríguez Mahecha 1998). La heterogeneidad de los paisajes de la región ha permitido el establecimiento de especies con una alta diversidad de hábitos y formas, las cuales difieren en cuanto a dieta, patrones comportamentales, de locomoción y de actividad diaria. 
Las sabanas inundables sobresalen por su riqueza hídrica, diversidad cultural, de paisajes y de ecosistemas terrestres y acuáticos que albergan distintas formas de vida hasta hace poco conocidas. Con un proceso histórico de ocupación y uso de sus recursos que ha generado diversos y controvertidos conflictos, es también una de los ecosistemas menos densamente pobladas del país, y al mismo tiempo uno de las que cuenta con un mayor grado de transformación en las últimas cuarenta años. El potencial petrolero en el subsuelo llanero ha sido en los últimas décadas la base de la economía, junto con actividades agropecuarias como ganadería, cultivos de pancojer, palma y arroz, entre otras (Correa et al., 2005).

En 1997 el Instituto Alexander von Humboldt (IAvH), formuló el proyecto "Biodiversidad y Desarrollo en Ecorregiones Estratégicas de Colombia - Orinoco", ocho años más tarde presentan el plan de acción en biodiversidad de la cuenca del Orinoco Colombia 2005 - 2015. Para ese propósito conformaron una mesa de trabajo interinstitucional integrada por la Corporación Autónoma Regional de la Orinoquia CORPORINOQUIA, la Corporación para el Desarrollo Sostenible de la Macarena - CORMACARENA, la Universidad de los Llanos, la Fundación Internacional del Trópico Americano - Unitrópico, la Pontificia Universidad Javeriana, la Fundación Omacha, la Fundación Horizonte Verde, la Agencia de Cooperación Alemana para el Desarrollo - GTZ, y WWF Colombia, coordinada por el Instituto Alexander von Humboldt.

Este trabajo presenta el primer diagnóstico para la región orinocense en donde se recopiló, sistematizó y analizó información para finalmente consolidar una visión más precisa y actualizada del estado de la región en términos de su biodiversidad. Se planteó y concertó un conjunto de líneas de acción, que buscan cubrir los vacíos de información y desarrollar las potencialidades regionales, para fortalecer el conocimiento, conservación y uso sostenible de la diversidad biótica y cultural. Así mismo, proponen una fase de seguimiento y evaluación que hace del plan un proceso permanente y dinámico que se va retroalimentando a medida que se avanza en la planificación. El fin último es que este documento fuese la ruta que recorran los involucrados e interesados en desarrollar el potencial de la biodiversidad en los próximos diez años en la cuenca del Orinoco (Correa et al., 2005).

En 2009, 2010 y 2011 se realizaron el segundo, tercer y cuarto taller binacional de la cuenca del Orinoco en Colombia donde se sentaron las bases del conocimiento actual y la actualización de la información existente, se definieron áreas prioritarias para la conservación y se presentaron las perspectivas y las iniciativas de trabajo en la región. De estos talleres se publican dos libros Biodiversidad de la cuenca del Orinoco tomo I y II (Lasso et al., 2010 y Lasso et al., 2011).

A partir del plan de acción de la biodiversidad 2005 2015 y los talleres Binacionales se desprende una serie de trabajos que se han publicado en ecosistemas de sabanas inundables en Colombia, principalmente en el departamento de Casanare.

La biodiversidad de las sabanas inundables presentes en el departamento de Casanare, ha sido estudiada por varios autores (Viña et al., 1997, Aldana-Domínguez et al., 2003, Fundación Puerto Rastrojo 2005, Fundación Terrapreta y WWF 2005, IAvH y Fundación Horizonte Verde 2006, Aldana-Domínguez y Ángel-Escobar 2007, Zamudio et al., 2008, Cortés et al., 2009, Ramírez et al., 2009, Urbano-Bonilla et al., 2009a, 2009b; León, 2011; Peñuela et al., 2011a; Peñuela et al., 2011b; Usma y Trujillo, 2011). A pesar de su importancia ecológica y socioeconómica, el departamento es uno de los más degradados del país, con mayor porcentaje de ecosistemas transformados $(19 \%)$ y sus ecosistemas están entre los menos representados en el Sistema Nacional de Áreas Protegidas (Arango et al., 2003; Lasso et al., 2010; Usma y Trujillo, 2011).

A continuación se reseña brevemente los estudios que se han realizado para los grupos de vertebrados focales (mamíferos, aves, anfibios, reptiles y peces) la flora y vegetación de las sabanas inundables en la Orinoquia Colombiana.

\section{Mamíferos}

Para la cuenca del Orinoco la información de mamíferos fue consolidada por Ferrer y colaboradores en 2009 registrando 318 especies. En Colombia, los estudios de diversidad de mamíferos han mostrado una alta riqueza de especies para los territorios de la Orinoquia registrando alrededor de 250 especies, de tal manera que esta región estaría representando cerca del $40 \%$ del total de mamíferos para el país (Hernández at al., 1984; Chaves y Arango 1998; Alberico et al., 2000; Correa et al., 2006; Ferrer et al., 2009; MatillaMeluk et al., 2009, Peñuela et al., 2011b).

Chaves y Arango (1998) reportan de manera preliminar 101 especies pertenecientes a 72 géneros, 26 familias y nueve órdenes para toda la cuenca del Orinoco. Sin embargo, las estimaciones de riqueza varían de acuerdo con el enfoque que se ha utilizado para el análisis: ecosistémico, de unidad de paisaje o de provincia biogeográfica (Correa et al., 2006), situándose entre 167 
(Alberico et al., 2000), y 210 especies (Correa et al., 2006). Ferrer et al., (2009), reportan un total de 183 especies de mamíferos para la región de los llanos, de las cuales el orden Chiroptera aporta 101 (55\%), seguida por Rodentia con 27 (14,75\%), Carnívora con 17 (9,3 $\%)$, Primates con 12 (6,5\%) y Didelphimorphia con 10 (5,5\%). Peñuela et al., (2011), reporta para las reservas naturales de la sociedad civil presentes en Arauca y Casanare 26 especies teniendo en cuenta exclusivamente medianos y grandes mamíferos.

Aunque en la región sólo se reportan dos especies de mamíferos endémicos (Aotusbrumbacki y Proechimysocconnelli), ésta se caracteriza por albergar las mayores concentraciones poblacionales de mamíferos del país (Rodríguez-Mahecha et al., 2006), con zonas de gran importancia como los humedales de Arauca y Casanare donde se agregan miles de chigüiros (Trujillo et al., 2011). Entre los estudios realizados para toda la cuenca del Orinoco (definida según Lasso et al., 2010), se destacan los concernientes a la ecología del chigüiro (Hydrochoerushydrochaeris) (Aldana et al. 2004, Vega 2004, Hernández-Camacho et al., 1983). Otras especies de roedores estudiadas son las del género Cavia, de las cuales existe una revisión taxonómica (Zuñiga 2002). Defler (2003), reporta 16 especies de primates, de las que se han adelantado investigaciones con énfasis en hábitat y poblaciones en algunas áreas, especialmente del piedemonte (Pinzón-Melo \& Baptiste - Ballera 2004). Además, existen datos sobre las subespecies y la distribución del venado cola blanca (Odocoileusvirginianus) (Rodríguez-Mahecha et al., 2006, González-Hernández 2001). En cuanto a los mamíferos acuáticos (Díazgranados-Pitti \& TrujilloGonzález 2004) han realizado estudios para delfines, manatíes (Castelblanco-Martínez y Bermúdez 2004) y nutrias (Carrasquilla y Trujillo 2004). Finalmente, el grupo más estudiado es el de los murciélagos, aunque la mayoría de estudios se concentran en la Sierra de la Macarena (Muñoz-Saba et al., 1997, Sánchez-Palomino et al., 1993), encontrándose sólo el estudio de Arias (1996) para todos los Llanos Orientales.

Según Usma y Trujillo (2011), los mamíferos del departamento del Casanare han sido mejor estudiados en comparación con los otros departamentos de la Orinoquia colombiana, debido a requerimientos de licencias ambientales que promueven la realización de inventarios en zonas de impacto. Suárez-Castro y Sánchez Palomino (2011) caracterizaron el ensamblaje de mamíferos en zonas de los municipios de San Luis de Palenque y Trinidad. A pesar de esto, la mayoría de estudios de impacto ambiental son aislados, no son de fácil acceso, y, en algunos casos, contienen información de baja confiabilidad (Ferrer et al., 2009).

\section{Aves}

Presentan una riqueza cercana a 1900 especies (Hilty y Brown 2001) y en la cuenca del Orinoco se ha registrado cerca de 1200 especies (Restrepo-Calle et al., 2010), de las cuales 877 se distribuyen en Colombia (Murillo-Pacheco 2005).

Se destacan estudios que se han realizado para identificar áreas claves para la conservación de las aves migratorias de la cuenca del Orinoco (Restrepo-Calle y Peña-Herrera 2005, Fierro 2009), la guía de las aves de los Llanos (McNish 2007) y el análisis binacional que identificó 21 áreas prioritarias para la conservación de las aves de la cuenca del Orinoco, tres de éstas presentes en el departamento del Casanare (Restrepo-Calle et al., 2010).

En el trabajo realizado Zamudio et al., (2011) en el departamento de Casanare, a partir de la integración de la información publicada, colecciones biológicas y observaciones de campo realizadas en los municipios de Hato Corozal, Orocué, Tauramena y Trinidad, registran una riqueza 507 especies para el departamento. Este registro incrementó en 29 especies el listado de aves del departamento, documentó la ampliación del rango de distribución geográfica de nueve especies amazónicas y logró identificar hábitats y áreas para la conservación regional, nacional e internacional de las aves en Casanare (Zamudio et al., 2011).

En el departamento se identificaron cuatro especies endémicas para los llanos (Brachygalba goeringi, Hypnelus ruficollis, Phacellodomus rufifrons y Phelpsia inornata), ocho especies con algún grado de amenaza a nivel nacional, siendo dos de estas aves migratorias (Contopus cooperiy Dentroica cerúlea) de importancia internacional ya que en la última década han disminuido su rango de distribución (Peñuela et al., 2011b; Zamudio et al., 2011).

La posición geográfica de los departamentos de Arauca y Casanare lo convierten en un sitio de transición entre los andes, la llanura orinocense y la fauna de la Amazonia, donde convergen especies típicas del piedemonte, zonas altas de los Andes, sabana y llanura inundable (Zamudio et al., 2011). Las aves constituyen un elemento estructural de los ecosistemas del Orinoco, prestan servicios ambientales como polinizadores, en el control de plagas, la fertilización de humedales, la dispersión de semillas, y como indicadores de la calidad ambiental y un elemento de atractivo turístico Zamudio et al., 2011).

\section{Anfibios y reptiles}

En la Orinoquia colombiana se reportan 108 especies de anfibios (Acosta-Galvis y Alfaro-Bejarano, 2011) y 
119 especies de reptiles (Chaves Santamaría 2006), los registros existentes en colecciones biológicas registradas en Colombia se remiten a las regiones aledañas al piedemonte llanero y a las inmediaciones de las riberas del río Orinoco, dejando de lado el paisaje de sabanas inundables que incluye los bosques de galería, matas de monte, bajos, bancos y esteros (AngaritaSierra en prensa).

En general, la información acerca de la herpetofauna para sabanas inundables en Colombia se encuentra consignada en un informe técnico sobre el complejo de humedales asociados a los ríos Caranal, Lipa y Cinaruco en el departamento de Arauca (Caro et al., 2006), los reportes de reptiles para el municipio de Trinidad y San Luis de Palenque (Cortés y Sánchez, 2010), la actualización realizada por Acosta-Galvis y Alfaro-Bejarano (2011) sobre el estado de conocimiento de los anfibios y reptiles del Casanare en los últimos cinco años, y los listados de la fauna y flora de las reservas naturales de la sociedad civil del Nodo Orinoquia de Resnatur (Peñuela et al., 2011).

Acosta-Galvis y Alfaro-Bejarano, (2011) reportan siete especies de anfibios que se encuentran amenazadas según UICN (2011) y dos según el libro rojo de anfibios de Colombia. De igual manera para reptiles se registra dos especies endémicas 13 bajo el criterios de explotación comercial CITES y siete especies amenazadas entre las que destacan Crocodilus intermedius, Podocnemis expansa y Podocnemis sunifilis, que requieren urgentes esfuerzos de conservación (Alfaro et al., 2011). El conocimiento acerca de estos grupos es muy limitado en sabanas inundables, por lo que se deben centrar esfuerzos en estos grupos.

\section{Peces}

La diversidad de peces en la Orinoquia ha sido estudiada en las cuencas de los ríos Meta (Sánchez et al., 1999), Arauca (Rugeles-Lugo et al., 2007), Bita (Maldonado-Ocampo 2000, Ortega-Lara 2005), Tuparro (Maldonado-Ocampo y Bogotá-Grégory 2007), Tomo (Maldonado-Ocampo et al., 2006), Mataven (Maldonado-Ocampo et al., 2009) y Orinoco (Galvis et al., 2007).

Estos estudios han sido compilados y complementados con nuevas especies posicionando la cuenca del Orinoco como la segunda región más diversa de Colombia con 658 especies, cerca del $46 \%$ de la riqueza íctica nacional (Maldonado-Ocampo et al., 2008)

En el trabajo realizado Villa-Navarro y colaboradores en 2011 en el departamento de Casanare, a partir del análisis de las bases de datos de colecciones científicas nacionales y extranjeras y muestreos estandarizados en los municipios de Tauramena (subcuenca del río Cusiana), Orocué (Subcuenca de los ríos Cravo Sur y Meta) Trinidad (Subcuenca del río Pauto), Hato Corozal y Paz de Ariporo (Subcuenca del río Casanare). Se registra 567 especies de peces agrupadas en 242 géneros, 41 familias y 10 órdenes. Esta riqueza representa el $86 \%$ de las 658 especies de la Orinoquia colombiana. Los órdenes Characiformes (263 spp.) y Siluriformes (202 spp.), tienen el mayor número de especies, mientras que las familias Characidae (167 spp.) y Loricariidae (64spp.) tuvieron la mayor representación específica.

Registran 29 especies de peces endémicas para la cuenca del río Meta; 11 especies con alguna categoría de amenaza, 232 con valor comercial (171 ornamental, 61 de consumo) y 58 especies migratorias. Se destacan seis nuevos registros para Colombia Apareiodon orinocensis, Melanocharacidium nigrum, Acestrocephalus ginesi, Rineloricaria caracasensis, Hypostomus shemicochliodon y Rhamphichthys apurensis; nueve especies se registran por primera vez en la cuenca Orinoco Steindachnerina bimaculata, S. dobula, Leporinus moralesi, L. subniger, Ammocryptocharax minutus, Hemigrammus tridens, Centromochlus reticulatus, Bunocephalus coracoideus y Vandellia cirrhosa. Esta riqueza posiciona al departamento de Casanare, como uno de los departamentos megadiversos de fauna íctica para Colombia (Villa-Navarro et al., 2011).

\section{Flora y Vegetación}

La diversidad florística de las sabanas inundables en Colombia ha sido estudiada recientemente y exististe pocas publicaciones al respecto. En trabajos realizados en de las reservas de la sociedad civil del nodo Orinoquia (Peñuela et al., 2011b) presentes en este ecosistema se reporta lo siguiente: en el municipio de Orocué en la reserva Palmarito se reportan 237 especies (Córdoba 2010) y en la finca Altamira 737 especies (Castro F. y Córdoba M. 2012) y en un trabajo realizado por Romero LP. y Castro F. 2012 en este mismo municipio se reportan 615 especies. En reservas de la sociedad civil en los municipios de Paz de Ariporo, Hato Corozal, Trinidad, San Luis de Palenque (Catro F. 2009; Castro F. 2011 y Peñuela et al., 2011b) reportan un total de 668 especies.

De acuerdo con los registros del trabajo realizado por Córdoba et al., (2011) en el departamento de Casanare, se registra un total 1479 especies de flora, distribuidas en 146 Familias (135 Magnoliophytas y 11 
Pteridophytas) y 659 Géneros. El predominio de las familias Poaceae, Cyperaceae y Fabaceae sobre las demás familias permite reconocer el tipo de ecosistema que impera en el departamento del Casanare, en donde la mayor parte del territorio corresponde a sabanas inundables con Poaceae, Cyperaceae y otras herbáceas que conforman asociaciones vegetales típicas de la región.

Para las sabanas colombianas existen diversas publicaciones de vegetación asociadas a diversidad (Fernández et al., 2010), ecología (Sarmiento, 1996; Baptiste y Ariza, 2008) y manejo (Rippstein et al., 2001; Correa et al., 2005; Peñuela et al., 2011), la literatura se encuentra muy dispersa y muestra escalas de información geográfica muy heterogénea, lo que dificulta la comparación entre estudios. Según Fernández et al., (2010) las sabanas inundables de la Orinoquia colombiana presentan vacíos de información, así como niveles bajos de muestreo y de conocimiento de la flora, debido en parte a la falta de inventarios florísticos y ecológicos a nivel regional.

Algunos de los trabajos más importantes que recopilan la mayor parte de información sobre la composición florística de los bosques de galería de la región de la Orinoquía en Venezuela son: el catálogo anotado e ilustrado de la flora vascular de los llanos de Venezuela (Duno de Stefano et al., 2007), particularmente los capítulos sobre los bosques (Aymard y González 2007) y los morichales (Fernández 2007). Mientras que en Colombia se pueden citar aproximaciones regionales en cuanto a tipos de vegetación a Cuatrecasas (1958), Holdrige et al., (1963), IGAC (1983), Salamanca (1984) y Van der Hammen y Rangel (1997); en cuanto a composición florística se encuentra una breve reseña por departamentos y municipios en Peñuela et al., (2011) y una revisión completa en Minorta (en prensa).

En cuanto a bosques de galería de la región de la Orinoquía, la mayor parte de información general sobre la estructura en se ha recopilado en trabajos Aymard y González (2007) y Fernández (2007) (particularmente sobre los morichales) en el lado venezolano. En Colombia, la compilación más actualizada fue presentada por Rangel (1992), pero desde entonces se han realizado más trabajos en la Orinoquía colombiana. Para una revisión más detallada del tipo y naturaleza de los trabajos realizados sobre flora y estructura de la vegetación en la región, véase los aportes de Correa et al., (2006), Fernández et al., (2010) y Córdoba et al., (2011).

En éste último trabajo, la gobernación del departamento, en alianza con la WWF, reportan algunos inven- tarios florísticos y evaluaciones ecológicas que son aportes valiosos al conocimiento de la Orinoquía y de Casanare. No obstante, el consenso de dicho trabajo es que aún hay muchas localidades que no han sido estudiadas, y aspectos en los cuales debe profundizar como la ecología, funcionalidad ecosistémica, fisiología, morfología y atributos vitales de las especies vegetales que son esenciales para fundamentar propuestas de restauración y conservación de los sistemas naturales. La única publicación que se encuentran tanto para bosques, como para sabanas, es la realizada adicional a la anterior en el Bloque de perforación exploratorio de hidrocarburos Cubiro llevada a cabo por el Instituto de Estudios Ambientales (IDEA) en alianza con AlangeEnergy Corp., en la que se analiza la composición de las comunidades vegetales, su estado de conservación y sus amenazas (Mora-Fernández, 2011).

\section{Limnologia}

Existen pocos estudios acerca de la composición y estructura de las diferentes comunidades hidrobiológicas con el fin de entender las funciones ecológicas que dentro de los sistemas acuáticos cumplen, como por ejemplo entender la transferencia de materia y energía que se da entre los diferentes eslabones de la cadena trófica que va desde los productores primarios como fitoplancton para sistemas lenticos, fitoperifiton para sistemas de aguas corrientes o lóticos hasta las macrofitas acuáticas sumergidas, emergentes y flotantes típicas de los sistemas acuáticos tropicales, y la transferencia de energía a niveles superiores de la cadena partiendo de los productores secundarios como el zooplancton, zooperifiton y la comunidad del necton (Peces) (Lasso et al., 2010; Rial et al., 2010).

En la Orinoquia colombiana se han realizado estudios que van desde investigaciones (pocos) efectuadas por la universidades de la región hasta estudios de consultoría (la mayoría). En general, los estudios de consultoría los realizan empresas petroleras, centrando su interés en las caracterizaciones bióticas y fisicoquímicas, y la evaluación y descripción de la calidad de los cuerpos de agua superficiales. Estos estudios técnicos no están disponibles para el público general dado al carácter privado de las empresas que los realizan (Lasso et al., 2010).

\section{Conservación y usos sostenible de la sabana inundable}

La Orinoquia colombiana, representa cerca del 50\% del territorio colombiano, donde encontramos, lagunas, esteros, sabanas y bosques inundables, que no se 
encuentra representado en las áreas de importancia internacional Ramsar y que son únicas en Colombia (Lasso et al., 2010).

En el Casanare desde hace ocho años se viene desarrollando un proceso para declarar un área protegida de carácter nacional en el municipio de Paz de Ariporo, al igual que estudios de planificación eco-regional y se han generado propuestas de áreas prioritarias para la conservación (ANH et al., 2007, WWF 1998, Phillips 2007, Lasso et al., 2010; Lasso et al., 2011; Usma y Trujillo 2011; Mora-Borrás 2011) de la biodiversidad en armonía con un desarrollo sostenible (Fondo FEN 1998, Correa et al., 2006, Rodríguez et al., 2009; Lasso et al., 2011; Usma y Trujillo 2011; Peñuela et al., 2011). Entidades gubernamentales y no gubernamentales han realizado convenios que propenden por la conservación de los humedales Colombianos. Un ejemplo es el esfuerzo de la organización internacional TheNatureConservancy (TNC) y el Sistema Administrativo Nacional de Parques Naturales (PNN), los cuales han identificado las áreas prioritarias de conservación en las Sabanas del Casanare. Sin embargo estos estudios no han sido publicados (Rodríguez, 2009). Al igual, la Gobernación de Casanare, WorldWildlifeFund Colombia (WWF Colombia), Fundación Omacha y el Instituto de Investigaciones en recursos biológicos Alexander von Humboldt $(\mathrm{I} A \mathrm{vH})$, realizaron un extenso estudio con el fin de priorizar algunas áreas del departamento para tener en cuenta en el momento de proponer áreas de conservación en el que se generó cartografía de caracterización física, social y biótica del Casanare (Lasso et al., 2010; Usma y Trujillo, 2011).

Por otro lado, en el Centro de Investigación del Agustín Codazzi (CIAF), en 2011 se desarrolló una tesis de grado en buscó proponer zonas Ramsar en Colombia a partir de la implementación de los sistemas de información geográfica (SIG). La tesis titulada "Propuesta de un área Ramsar en las Sabanas Inundables del Departamento de Casanare-Colombia, aplicando herramientas de sistemas de información geográfica", para analizar y modelar las capas temáticas de los criterios seleccionados por los autores, usados para la declaración Ramsar, definiendo zonas de alto valor de importancia y necesidad de conservación (Mora y Borrás, 2011).

El Nodo Orinoquia de la Asociación Red Colombiana de Reservas Naturales de la Sociedad Civil (RESNATUR), coordinado por la Fundación Horizonte Verde, ha venido trabajando desde el año 2007 en el paisaje de sabana inundable en el departamento de Casanare, con un enfoque de conservación producción, estable- ciendo nuevas reservas que contribuyan a conservar parches de ecosistemas de sabana, realizando inventarios de flora y fauna, elaborando e implementando planes de manejo; generando e implementado alternativas para mejorar la productividad de la ganadería de cría, explotación que ha ocupado el territorio en los últimos 500 años.

A diciembre de 2012 se cuenta con 10 Reservas asociadas al Nodo, con un área total de 22.120 ha en paisaje de sabana inundable; 457 especies de plantas identificadas, 212 especies de aves; 23 especies de mamíferos; 33 especies de peces y 17 especies de entre anfibios y reptiles. (Peñuela et al., 2011).

Existen varios esfuerzos de diferentes entidades para el desarrollo de propuestas para la definición y conservación de áreas que se encuentran fuera de la protección nacional ambiental y regional y que a criterio de estas entidades o personas son de gran importancia a nivel nacional e internacional en ecosistemas con alta integridad ecológica como es el caso de las sabanas inundables.

\section{Herramientas de gestión y planificación}

La mayoría de los documentos que tratan temas de gestión y planificación ambiental son informes técnicos, planes de manejo ambiental, planes de ordenamiento de cuencas, políticas ambientales para la Orinoquia, entre otros.

La Corporación autónoma Regional de la Orinoquia (Corporinoquia), se preocupó por el futuro de la región ya que esta atraviesa por un proceso de transformación económico, social y ecológico sin precedentes. El grado de incertidumbre sobre el resultado de este proceso de transformación es alto, como consecuencia de carencia de información y el desconocimiento sobre el funcionamiento de los sistemas ecológicos y sociales en escenarios de cambio global dado a la debilidad institucional y la falta de un acuerdo social sobre el futuro de la región. En consecuencia, CORPORINOQUIA en convenio con la Universidad de Los Andes, Facultad de administración publican en octubre de 2009 el libro "La mejor Orinoquia que podemos construir, elementos para la sostenibilidad ambiental del desarrollo" (Andrade et al., 2009) en donde abarcan temas como la naturaleza de la Orinoquia, visiones en la Orinoquia colombiana, transformación productiva de los ecosistemas de la Orinoquia, oportunidades de desarrollo dentro de los límites de funcionamiento de los ecosistemas, CORPORINOQUIA como gestión ambiental en transición, gestión de conservación y realidades financiaras de la gestión ambiental. 


\section{Educación ambiental}

La educación ambiental está orientada a lograr que los seres humanos tomen conciencia de su papel activo a favor del manejo adecuado del ambiente y acciones por medio de la introducción de cambios en sus comportamientos cotidianos en actividades laborales y sociales (Bermúdez 2008).

En los escenarios rurales colombianos, la relación con el ambiente es directa, estas comunidades dependen de fuentes naturales de agua, madera, proteína animal, producción pecuaria local para el autoconsumo y/o para la generación de ingresos económicos, en algunos casos, a partir de la medicina tradicional derivada de elementos de la biodiversidad local (Ramírez y RoaLópez, 2011).

Con algunas comunidades rurales de la sabana inundable del Casanare se han desarrollado "Programas de Conservación de Fauna Silvestre y Recursos Naturales", dirigidos específicamente a las veredas de Mariara y Guariamena del municipio de Orocué y en las veredas Corea y Mundo Nuevo del municipio de Maní, entre el 2008 y 2009 por la empresa Ávila\& Miranda, 2011. En esta última vereda (Mundo Nuevo) se desarrolla la segunda fase del Programa entre el segundo semestre de 2010 y primer semestre de 2011.

De la misma forma se ha desarrollado con la vereda Surimena del municipio de Orocué entre los años 2010 y 2011 el Programa de Evaluación, Seguimiento y Conservación del Área Ambientalmente Sensible El Garcero, el cual se ubica en la Finca Banco Largo de esta misma vereda, enfoca el desarrollo de la educación ambiental a la identificación, caracterización y consolidación de compromisos y participación comunitaria frente a la preservación de esta importante área (Ávila y Miranda, 2011).

A través de los programas ejecutados se han identificado diferentes problemáticas que vienen afectando los ecosistemas, recursos naturales y la salud humana desde la perspectiva de las comunidades, como lo son: la contaminación de los cauces por actividades petroleras y arroceras, la pérdida de hábitat por monocultivos de arroz, palma africana y el cambio de las dinámicas hídricas. Las problemáticas locales como caza, deforestación y desecación de la sabana inundable, han acarreado la disminución y la calidad de los cuerpos de agua, pérdida de áreas boscosas y naturales, y por ende de fauna silvestre (Ávila y Miranda, 2001; Ramírez y Roa-López, 2011).

\section{Resultados}

\section{Tipos de publicaciones}

Incluimos un total de 157 publicaciones. La mayor parte corresponde a artículos y resúmenes de trabajos presentado en eventos (19\%), seguido por libros $(18 \%)$, capítulo de libro $(16 \%)$ y tesis e informes técnicos (14\%). El 53\% del número de publicaciones corresponde a literatura publicada oficialmente y el restante $47 \%$ a literatura gris (que incluye los informes técnicos, tesis y resúmenes en eventos), como se aprecia en la Figura 2.

Dado que el $47 \%$ de las publicaciones corresponde a literatura gris, implicala dificultad que existe para acceder a la información, la poca divulgación de la misma y un impacto mínimo en la población en general. Es evidente que existen motivos para no someterse alos procesos de evaluación que exigen los documentos a publicarse que mejora la calidad de la información. Para la toma de decisiones, con este tipo de información, se dificulta llevar a cabo procesos de monitoreo, para medir los cambios en el tiempo, respecto a la dinámica, problemáticas y restauración de las sabanas inundables.

Por otro lado el 53\% corresponde a literatura formalmente publicada, lo que indica mayor impacto y poder divulgativo en general. Se evidencia interés en generar información de calidad, lo que favorece y brinda herramientas para los tomadores de decisiones.

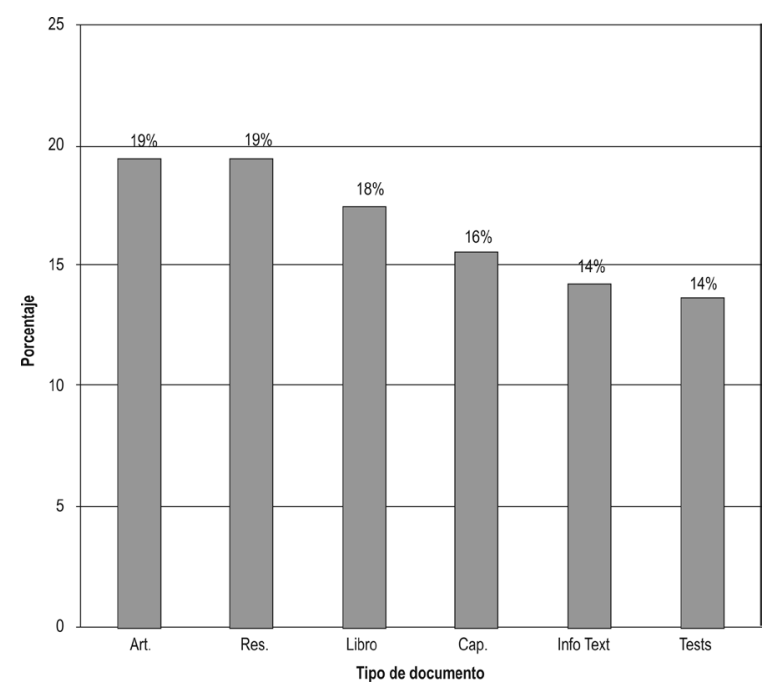

Figura 2. Tipo de documento. Art: Artículo; Info. Téc.: Documento técnico; Cap: Capitulo de Libro; Res: Resumen de un trabajo presentado en un evento. 


\section{Categorías temáticas}

El mayor porcentaje de las publicaciones corresponde a estudios de ecología, flora y fauna $(68 \%)$, seguido por conservación y usos sostenible $(27 \%)$, educación ambiental ( $3 \%)$ y gestión y planificación $(2 \%)$, como se aprecia en la Figura 3.

Los estudios en flora fueron los mejor representados (38\%), seguidos de mamíferos y peces $(18 \%)$ y aves $(12 \%)$, y los grupos menos representados son losanfibios $(6 \%)$ y los reptiles $(8 \%)$, como se aprecia en la Figura 4.

Los estudios de ecología, flora y fauna presentaron mayor número de publicaciones (105 publicaciones), de las cuales el $60 \%$ son publicaciones formales lideradas por investigadores independientes, instituciones nacionales tales como la Universidad Nacional de Colombia, Instituto de Investigaciones en recursos biológicos Alexander von Humboldt (IAvH), Instituto Geográfico Agustín Codazzi (IGAC), Instituto de hidrología, meteorología y estudios ambientales de Colombia (IDEAM), Corporinoquia, Gobernación de Casanare, Parques Nacionales Naturales y organizaciones sin ánimo de lucro nacionales e internacionales: Fundación Horizonte Verde (FHV), WorldWildlifeFund Colombia (WWF), TheNatureConservancy (TNC); con el fin de generar conocimiento de su territorio para una mejor gestión del mismo.

De las publicaciones enfocadas a conservación y uso sostenible, el 35\% de estas son formales. En educación ambiental, (3 publicaciones todas formales); y gestión y planificación ( 5 publicaciones y solo una formal). Laspocas herramientas que se generan para la toma de decisiones y el impacto divulgativo es mínimo. Se deben enfocar esfuerzos en realizar más ejercicios de

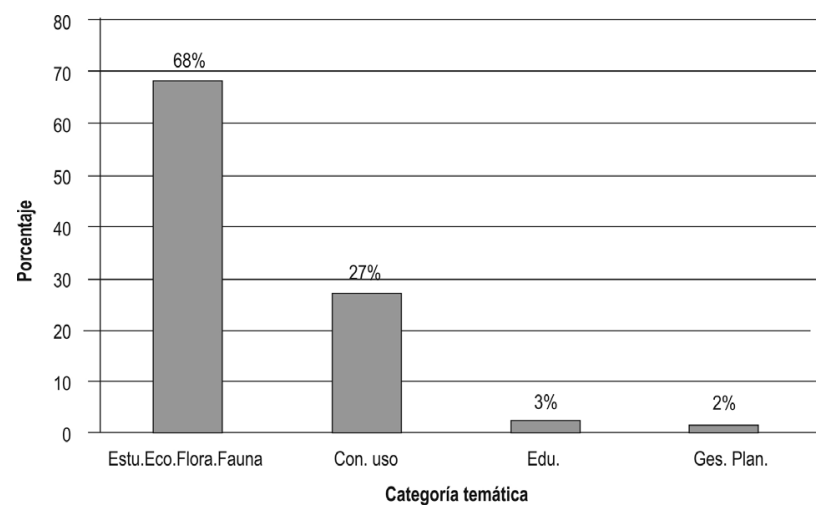

Figura 3. Publicaciones por categoría temática. Estu.Eco. Flora.Fauna: Estudios en ecología, flora y fauna; Con. Uso: Conservación y Uso sostenible; Edu.: Educación Ambiental; Ges. Plan: Gestión y Planificación.

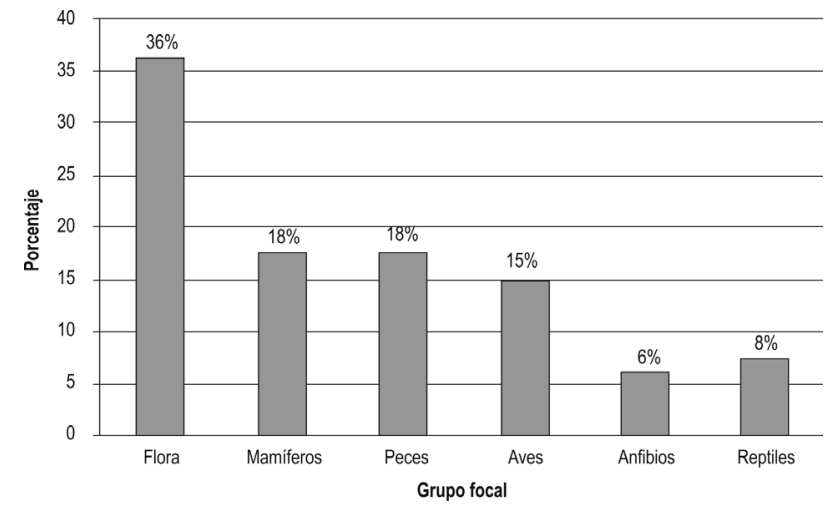

Figura 4. Publicaciones por Grupo Focal.

gestión y planificación del territorio, divulgarlos, para obtener mayor impacto

Los estudios de ecología, flora y fauna son los que normalmente tienen mayor número de publicaciones asociadas, porque es importante entender los procesos que ocurren en el sistema cuando se quiere o se ha realizado alguna intervención y para el monitoreo de los mismos. Sin embargo, se han dejado a un lado los trabajos de conservación y uso sostenible, donde el hombre y sus procesos de explotación y producción son lo fundamental.Se sabe muy poco de los impactos generados por los sistemas de producción que se desarrollan en las sabanas inundables, y son pocas las alternativas que existen para mejorar la productividad favoreciendo la biodiversidad.

Los trabajos de educación ambiental realizados con las comunidades han sido poco documentados. Muchos de ellos rara vez incluyen a la gente local, lo que hace difícil que las comunidades se sientan incluidos, se apropien de sus recursos y los valoren.

En cuanto a los trabajos para la gestión y la planificación del territorio, herramienta fundamental para el ordenamiento territorial, la mayoría son informes técnicos y son pocos los ejercicios de gestión y planificación publicados.

Ahora bien, en cuanto a los grupos focales, la flora y vegetación es la que mejor se ha estudiado, dado a que es un recurso fundamental para los habitantes de la región. Es el refugio de todas las especies que habitan en las sabanas inundables. Su conocimiento es esencial para fundamentar propuestas de restauración y conservación de los sistemas naturales (Córdoba et al., 2011). Se han realizado esfuerzos valiosos para incrementar el conocimiento en este grupo, sin embargo no han sido suficientes. 
En general se considera que hacen falta estudios mejor estructurados, que tengan en cuenta las sabanas, porque existe un gran vacío en cuanto a gramíneas, poaceas y demás plantas que allí se encuentran. La mayoría de los estudios están enfocados a los bosques de galería.

Otro grupo que presentó un número de estudios representativo son los mamíferos. Es un grupo de alta importancia biológica, cultual y económica en los paisajes donde se combinan zonas naturales y áreas utilizadas para cultivos y ganadería. Los mamíferos exponen una gran diversidad de hábitos y formas involucradas en diferentes procesos ecosistémicos y aportan una considerable biomasa animal específica, como recurso para las comunidades locales. Cumple un papel importante en procesos de dispersión de semillas, polinización y control de plagas (Alberico et al., 2000; Soriano et al., 2002, Rodríguez-Mahecha et al., 2006, Restrepo-Calle et al., 2010; Kunz et al., 2011; Peñuela et al., 2011b). Este grupo presenta especies carismáticas, que han recibido especial atención, debido al grado de amenaza a las que están sometidos por las diversas actividades humanas en los ecosistemas acuáticos. Se ha dejado a un lado grupos igual de importantes, pero más conspicuos, como los roedores y murciélagos, de los cuales el conocimiento para la Orinoquia es mínimo.

Los peces, son un grupo mejor estudiado para las sabanas inundables. Hasta mediados de la década del 2000 el conocimiento sobre peces, con distribución en el área los departamentos de Arauca y Casanare, se limitaba al desarrollo de algunos trabajos en el marco de estudios de impacto ambiental; no obstante, el levantamiento sistemático que se ha venido realizando sobre la ictiofauna, que se distribuye en los ríos que drenan por los departamentos de Arauca y Casanare, desde el año 2006 ha incrementado significativamente el número de especies registradas para estos departamentos (Rugeles-Lugo et al., 2007; Urbano-Bonilla et al., 2009b; Villa-Navarro et al., 2011).

En años recientes se han extendido estos estudios, con el fin de abarcar las regiones que incluyen las partes medias y bajas de las cuencas y los ecosistemas acuáticos asociados a sabanas inundables con preferencia en el departamento de Casanare (Urbano-Bonilla et al., 2009 datos no publ., Preciado 2011 datos no publ., Villa-Navarro et al., 2011). Dado que gran parte de las especies distribuidas en las cuencas de los departamentos de Arauca y Casanare tienen importancia, ya sea como especies de consumo u ornamental. El incremento en el conocimiento sobre la biología básica de estas especies es fundamental y se deben realizar esfuerzos en el departamento de Arauca.
Las aves han sido especialmente atendidas, porque son útiles para diseñar e implementar políticas de conservación y manejo de ecosistemas y hábitats. Aprtan información técnica para la identificación de comunidades que necesitan protección e información científica, para el desarrollo de estudios en biogeografía, sistemática, ecología y evolución (Murillo-Pacheco 2005; Restrepo-Calle y Peña-Herrera 2005; Mc Nish 2007; Restrepo-Calle et al., 2010; Peñuela et al., 2011b; Zamudio et al., 2011).

Caso contrario ocurre con los anfibios y los reptiles, que son tal vez uno de los ensambles menos conocidos en el país para las sabanas inundables. Existen estudios de algunos grupos proporcionando el poco conocimiento de la composición, distribución y biología del ensamble de anfibios y reptiles que tiene las sabanas inundables (Dunn 1942-1946; Medem 1950-1980; Stebbins y Hendrickson 1959-1960; Pyburn 1960-1980; Lynch 1982-2012; Ardila y Ruíz 1980-1997; Suárez y Rueda, 2002; Campbell y Lamar, 2002; Rueada-Almoacid et al., 2007; Cáceres-Andrade y Urbina-Cardona, 2009; Acosta-Galvis y Alfaro-Bejarano, 2011).

\section{Variación temporal}

Registramos publicaciones relacionadas a sabanas inundables de la Orinoquia colombiana desde el año 1964; periodo en el cual se presentó una sola publicación. La tendencia permanecióconstante, oscilando entre una y 6 publicaciones hasta el 2006, a partir de aquí se incrementa a 9 publicaciones. En el 2008 se presentaun ascenso a 16 publicaciones, manteniéndose así hasta el 2011 donde prácticamente se duplica con 31 publicaciones, reduciéndose a 20 hasta Noviembre de 2012, como se aprecia en la Figura 5a.

Observamos en el análisis por décadas, que la mayor contribución al conocimiento de las sabanas inundables de la Orinoquia colombiana se presenta en dos décadas: finalizando la década 1990-2000, donde se produjo el 39\%; y la década 2000-2010 donde se ha producido el $44 \%$ de la información generada para el país, como se aprecia en la Figura 6b.

\section{Departamentos}

Las sabanas inundables en la Orinoquia colombiana están presentes en dos departamentos y se reportaasí: para Casanare y Arauca 63 publicaciones (41\%), 74 publicaciones solo para Casanare $(48 \%)$ y 17 publicaciones solo para Arauca (11\%). Se evidencia mayores esfuerzos para trabajar en Casanare, presentándose un vacío de información en las sabanas inundables de Arauca, como se aprecia en la Figura 6. 

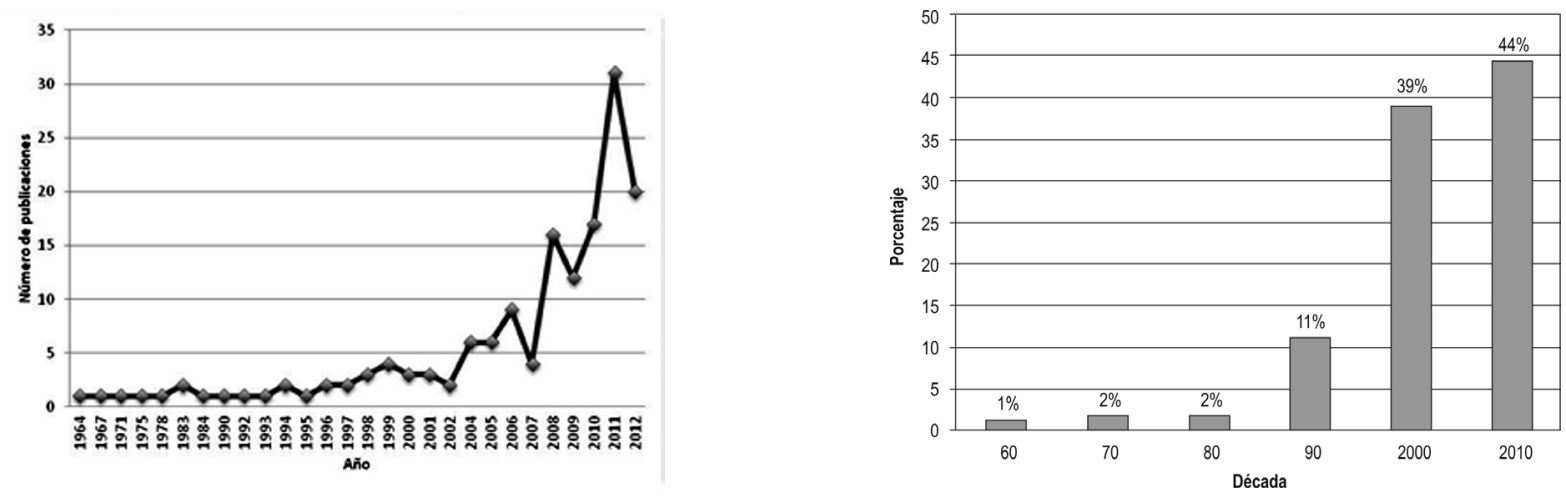

Figura 5 a) y b). Comportamiento temporal del número de publicaciones. a: Distribución por años; b: Distribución por década.

\section{Discusion}

Este análisis nos confirma la hipótesis que teníamos sobre la poca información disponible que existe para el paisaje de sabana inundable y recopila la existente. Es necesario mencionar que algunas referencias de literatura gris no fueron consultadas debido a la dificultad de acceder a ellas. Sin embargo, hicimos un ejercicio muy juicioso y creemos que el análisis que realizamos presenta algunos de los vacíos de información que se encuentran en la región y en los cuales deben iniciarse trabajos de investigación.

La revisión y análisis permite visualizar que existen algunos grupos más estudiados que otros y que es necesario realizar investigaciones más completas que cobijen la diversidad que se expresa en las sabanas inundables.

La estacionalidad es un factor determinante en la dinámica hidrológica de la de las sabanas inundables. Es fundamental empezar a trabajar sobre el servicio de regulación hídrica, su importancia en la dinámica y los procesos ecológicos de las sabanas inundables orinocenses.

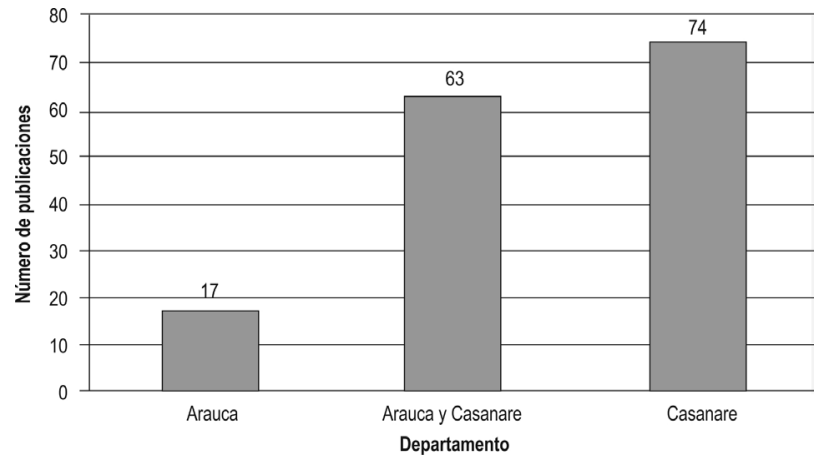

Figura 6. Número de Publicaciones por Departamento
Son loables los esfuerzos realizados por los propietarios de las Reservas Naturales de la Sociedad Civil, para conservar parches de ecosistemas de sabana inundable, pero es necesario prestar más atención al mejoramiento de la productividad ganadera e incentivos. Es la ganadería la actividad económica de predilección en la región, y es necesario generar alternativas viables para evitar la transformación de las sabanas por pastos introducidos, o por otras explotaciones agrícolas como el arroz y la palma de aceite.

Importante que los municipios de sabana inundable en los departamentos de Arauca y Casanare, utilicen las diversas herramientas de conservación, para proteger parches de ecosistemas de sabana inundable como patrimonio ecológico municipal. Así mismo que destinen recursos para generar mayor conocimiento, y articulen acciones con las empresas petroleras para minimizar los impactos negativos que puedan generarse sobre los recursos naturales, ecosistemas, flora y fauna de la sabana inundable.

Se evidencia la necesidad de abordar los temas de manejo de estos ecosistemas de manera integral, que no intente controlar la naturaleza para explotar todo su potencial, sino por el contrario, a partir del dialogo con los diferentes actores, se diseñen planes y estrategias que permitan aprovechar los recurso y servicios que necesite la sociedad, de una manera sustentable, que sea garantía para ser aprovechados por las generaciones futuras.

Es necesario profundizar en el conocimiento, conservación y uso de la sabana inundable de la Orinoquia colombiana, porque es un ecosistema estratégico, para la dinámica hidrológica, del tercer sistema ribereño más importante del mundo: La cuenca del Orinoco. 


\section{Referencias}

Acosta-Galvis AR, Ceñaris JC, Rojas-Runjaic F, Ruaño-Pinzón DR. 2010. Anfibios y Reptiles. 158-289p. En: Lasso CA, JS Usma, F Trujillo, A. Rial (eds.). 2010. Biodiversidad de la Cuenca del Orinoco: bases científicas para la identificación de áreas prioritarias para la conservación y uso sostenible de la diversidad. Instituto de Investigación de Recursos Biológicos Alexander von Humboldt, WWF Colombia, Fundación Omacha, Fundación La Salle e Instituto de Estudios de la Orinoquia (Universidad Nacional de Colombia). Bogotá, D. C., Colombia. 609 pp.

Acosta-Galvis Y,. Alfaro-Bejarano JP. 2011. Anfibios del Casanare. Pp. 138-151. En: Usma, J.S., y F. Trujillo (Editores). 2011. Biodiversidad del Casanare: Ecosistemas Estratégicos del Departamento. Gobernación de Casanare - WWF Colombia. Bogotá D.C. $286 p$

Aldana-Domínguez J, Ladino E. 2006. El caso del chigüiro (Hydrochoerushydrochaeris). pp 95-97. En: Correa H.D., S.L. Ruiz \& L. M. Arévalo (Eds). 2006. Plan de Ac- ción en Biodiversidad de la cuenca del Orinoco-Colombia/ 2005- 2015-Propuesta técnica. Bogotá D.C. Corporinoquia, Cormacarena, IAvH, Unitrópico, Fundación Oma- cha, Fundación Horizonte Verde, Universidad Javeriana, Unillanos, W WF-Colombia, GTZ-Colombia, Bogotá, Colombia. 330 p.

Aldana-Domínguez J, Forero, Betancur J, Cavelier J. Dinámica y estructura de la población de chigüiros (Hydrochaerishydrochaeris: Rodentia, Hydrochae- ridae) de Caño Limón, Arauca, Colombia. Caldasia. ;24(2):445-458.Aldana-Domínguez J, Vieira-Muñoz I, Ángel- Escobar DC. (Editoras). 2007. Estudios sobre la ecología del chigüiro (Hydrochoerushydrochaeris), enfocados a su manejo y uso sostenible en Colombia. Instituto Alexander von Hum- boldt. Bogotá, D.C., Colombia. 188p.

Alfaro JP, Acosta-Galvis, Vejarano M. 2011. Reptiles del Casanare. Pp. 152-167. En: Usma, J.S., y F. Trujillo (Editores). 2011. Biodiversidad del Casanare: Ecosistemas Estratégicos del Departamento. Gobernación de Casanare - WWF Colombia. Bogotá D.C. $286 p$

Alberico M, Cadena A, Hernández-Camacho J, Muñoz-Saba Y. Mamíferos (Synapsida:Theria) de Colombia. Biota Colombiana.; 1(1):43-75.

Allan JD, Flecker A. Biodiversity Conservation in Running Waters. Bioscience. 1993;43(1):32-43.

Andrade G, Castro LG, Duran A, Rodríguez M, Rudas G, Uribe E, Wills E. 2009. La mejor Orinoquia que podemos construir. Universidad de Los Andes. Bogotá, Colombia.

Ávila RC, Miranda LM. 2011. La Educación Ambiental como herramienta en la conservación de la Orinoquia. Pp. 246-257. En: USMA, J.S., y F. TRUJILLO (Editores). 2011. Biodiversidad del Casanare: Ecosistemas Estratégicos del Departamento. Gobernación de Casanare - WWF Colombia. Bogotá D.C. 286p.

Bayley PB. Understanding large river-flooplain ecosystems.BioScience. ;45:153-158

Bermúdez O. (Ed.) 2008. Memorias de los foros ambientales 20062007 realizados por la red temática de educación ambiental, en el marco de la década de la educación para el desarrollo sostenible propuesta por la UNESCO.
Botero P. (Ed.). Paisajes fisiográficos Orinoquia-Amazonia (ORAM) Colombia. Análisis Geográficos. ;27-28. 373 p.

Castro F. 2009. Caracterización de las reservas de la sociedad civil; la Esperanza y La Gloria en el municipio de paz de Ariporo. Informe técnico. Convenio de Cooperación Interinstitucional celebrado entre TheNatureConservancy, Asociación Red de Reservas de la Sociedad Civil-Resnatur y Fundación Horizonte Verde. 108p.

Castro F. 2011. Informe técnico. Caracterización de 6 reservas de la sociedad civil en el departamento de Casanare: La palmita (Trinidad), San José (Paz de Ariporo), La macarena (Paz de Ariporo)Agua verde (Hato Corozal), las delicias (San Luis) y Campo alegre (San Luis). Fundación Horizonte Verde. 200p.

Castro F, Córdoba MP. 2012. Caracterización de los ecosistemas en el predio Altamira, propiedad de aceites manuelita - Orocué, Casanare. Informe técnico. TNC-Manuelita. Bogotá D.C. Colombia. 103p.

Castro F, Peñuela RL. Caracterización de usos de la biodiversidad e identificación de opciones de manejo de recursos de la biodiversidad en el resguardo indígena caño mochuelo, Casanare. Plan de Acción en biodiversidad de la cuenca de la OrinoquiaColombia 2005-2015. Instituto de investigación de recursos biológicos Alexander von Humboldt IAvH) y Fundación Horizonte Verde. 16p.

Chaves HE, Santamaría M. (eds.) 2006. Informe Nacional sobre el avance en el conocimiento y la información de la Biodiversidad 1998-2004. Instituto de Investigaciones de Recursos Naturales Alexander Von Humboldt. Bogotá D.C. Colombia 2 Tomos.

Córdoba MP. 2010. Caracterización florística de la reserva natural de la sociedad civil PalmaritoOrocué-Casanare. Informe técnico. Fundación Omacha. Bogotá, D.C. 14p.

Córdoba CA, Ahumada JA, Pradilla G. 2011 Págs 329-415. Relaciones comunidad-fauna y educación ambiental en el Bloque Cubiro. En: LEÓN SICARD, T. (Editor). 2011. Mamíferos, Reptiles y Ecosistemas del Bloque Cubiro (Casanare): Educación Ambiental para la Conservación. Instituto de Estudios Ambientales Universidad Nacional de Colombia. AlangeEnergy Corp. Bogotá. 432pp.

Correa HD, Rioz SL, Arévalo LM. (eds.). 2005. Plan de Acción de Biodiversidad de la Cuenca del Orinoco - Colombia 2005-2015. Propuesta técnica Bogotá D.C. Corporinoquia. Cormacarena. $\mathrm{IAvH}$, Unitropico, Fundación Omacha, Fundación Horizonte Verde, Universidad Javeriana, Unillanos, WWF-Colombia, GTZColombia, Bogotá Colombia. 330p

Fierro K. 2009. Aves Migratorias de Colombia. Pp 63-75. En: Naranjo, L.G. y J.D. Amaya (eds.). Plan Nacional de las Especies Migratorias. Diagnostico e identificación de acciones para la conservación y manejo sostenible de las especies migratorias de la biodiversidad en Colombia. Ministerio de Medio ambiente, vivienda y DesarroIlo territorial y WWF Colombia. Bogotá D.C. Colombia.

Foley JA, Botta A, Coe MT, Costa MH. 2002. El niño-Southern Oscillation and the climate, ecosystems and rivers of Amazonia. Global Biogeochem. Cycles 16: Art. 1132

Fundación Puerto Rastrojo. 2005. Caracterización ecológica rápida corregimiento la Hermosa, municipio de Paz de Ariporo, departamento de Casanare, Colombia. WWF, Colombia y Unidad 
Administrativa Especial del sistema de Parques Nacionales Naturales (Bogotá Colombia). Informe Final. Bogota, Colombia.

Galvis G, Mojica Jl, Provenzano F, Lasso C, Taphord D, Royero R. et al. 2007. Peces de la Orinoquia Colombiana con enfasis en especies de interés ornamental. En: Sanabria-Ochoa, A.I., P. Vistoria.Daza y I.C. Bentrán (Eds.). 2007. Ministerio de Agricultura y Desarrollo Rural, INCODER, Universidad Nacional de Colombia - Departamento de Biología - Instituto de Ciencias Naturales. Bogotá, Colombia. 425p.

Gossen D. Geomorfología de los Llanos Orientales. Revista ACCEFYN, 1964;12(46):129-140.

Gossen D. 1971. Physiography and soils of the Llanos Orientales, Colombia. Sol Offsetdruk, Amsterdam: Publications of the Internacional Institute for Aerial Survey and Herat Sciences (ITC) - Enschede - The Netherlands. Series B Numer 64.

Hernández-Camacho J, Pachón JE, Rodríguez JV. 1983. Evaluación de las poblaciones de chigüiro (Hydrochae- rishydrochaeris) en los hatos Brasilia, Guamito, La Aurora, La Borra, El Danubio, La Veremos, y Mapurisa, municipio de Hato Corozal, Casanare. Informe presentado a Instituto Nacional de los Recursos Naturales Renovables y del Am- biente-INDERENA. Bogotá. Colombia. 48p.

Hilty S, Brown W. 2001. Guía de las aves de Colombia. ABC. Universidad del Valle, SAO. Bogotá. Colombia. 1030p.

Horn MH, Correa SB, Parolin P, Pollux BJA, Anderson JT, Lucas C, et al. Seed dispersal by fishes in tropical and temperate fresh waters: The growing evidence. Acta Oecologica. 2011;37(6):561-577.

Huber O, Alarcón C. 1988. Mapa de vegetación de Venezuela. 1:2.000.000. MARNR, The NatureConservancy, Caracas. 1p.

IGAC. 1983. Atlas Regional Orinoquia-Amazonia. Bogotá, D. C.: Instituto Geográfico Agustín Codazzi.

IGAC. 1983. Mapa de bosques. Departamento de Boyacá - Meta - Intendencias de Arauca - Casanare - Comisariade/Vichada. Bogotá, D. C.: Instituto Geográfico Agustín Codazzi.

IGAC. 1993. Suelos departamento de Casanare. Bogotá, D. C.: Instituto Geográfico Agustín Codazzi.

IGAC. 2002. Geomorfología y dinámica fluvial de un sector de los ríos Casanare, Cusiana, Manacacías y Pauto. Bogotá D. C.: Instituto Geográfico Agustín Codazzi, Oficina CIAF.

Junk WJ, Bayley PB, Sparks RE. The Flood pulse concept in riverfloodplain systems. En Dodge DP (Ed.) Proc. Int. Large River Symposium.Can. Spec. Publ. Fish.Aquat. Sci. 1989; 10-127.

Junk WJ, Watzen KM. 2004. The flood pulse cpncept: new aspects, approaches and applications -An update. En Welcomme RL, Petr T (Eds.) Proc. 2nd Int. Symp. Management of Large Rivers for Fisheries.Volume II. RAP Publication 2004/17. FAO Regional Office for Asia and the Pacific. Bangkok, Thailand. 117140pp.

Lasso CA, Usma, Trujillo F, Rial A. (Editores). 2010. Biodiversidad de la cuenca del Orinoco: bases científicas para la identificación de áreas prioritarias para la conservación y uso sostenible de la biodiversidad. Instituto de Investigación de Recursos Biológicos Alexander von Humboldt, WWF Colombia, Fundación Omacha, Fundación La Salle e Instituto de Estudios de la Ori- noquia (Universidad Nacional de Colombia). Bogota, D.C, Colombia. 609 pp.

Lasso CA, Rial A, Matallana C, Ramírez W, Señaris C, Díaz-Pulido A, et al. (Editores). 2011. Biodiversidad de la Cuenca del Orinoco; II. Áreas prioritarias para la conservación y uso sostenible. Instituto de Investigación de Recursos Biológicos Alexander von Humboldt, Ministerio de Ambiente, Vivienda y Desarrollo Territorial, WWF Colombia, Fundación Omacha, Fundación La Salle e Instituto de Estudios de la Orinoquia (Universidad Nacional de Colombia). Bogotá, D. C., Colombia. 304 pp.

León Sicard T. (Editor). 2011. Mamíferos, Reptiles y Ecosistemas del Bloque Cubiro (Casanare): Educación ambiental para la conservación. Instituto de Estudios Ambientales Universidad Nacional de Colombia, AlangeEnergy Corp. Bogotá. 432pp.

William M, Lewis Jr. 2008. Physical and chemical features of tropical flowing waters.En Dudgeon D. (Ed.) Tripical Stream Ecology. Elservier. Amsterdam, Holanda. pp. 1-21

Londoño-Arango M. 2008. Caracterización florística de la reserva privada Palmarito Casanare (municipio de Orocué, Casanare). Omacha, fundación Palmarito, WWF. Cali Colombia. 125p.

Maldonado-Ocampo JA. 2000. Peces de Puerto Carreño: Lista ilustrada. Fundación Omacha, Bogotá. 87pp.

Maldonado-Ocampo JA, Usma-Oviedo JS. 2006. Estado del Conocimiento sobre peces dulceacuicolas en Colombia. Tomo II. 174194p. En: Chaves, M.E. y M. Santamaria (Eds.) 2006. Informe sobre el avance en el conocimiento y la información de la Biodiversidad 1998-2004. Instituto de Investigaciones de Recursos Biológicos Alexander Von Humboldt. Bogotá D.C. Dos Tomos.

Maldonado-Ocampo JA, Bogotá-Gregory JD. 2007. Peces. 237245pp. En: Villarreal-Leal H. Y Maldonado-Ocampo J.A. (Comp.). Caracterización Biológica del Parque Nacional Natural el Tuparro (Sector NE), Vichada, Colombia. Instituto Alexander Von Humboldt. Bogotá D.C., Colombia. 286pp.

Maldonado-Ocampo JA, Vari R, Usma-Oviedo . Checklist of the Freshwater Fishes of Colombia. Biota Colombiana. ;9(2):143-237.

Maldonado-Ocampo JA, Bogotá-Gregory JD, Villa-Navarro FA. 2009. Peces. 171-181p. En: Villareal-Leal H., M. Álvarez-Rebolledo, M. Higuera-Díaz, J. Aldana-Domínguez, J.D. Bogotá-Gregory, F.A. Villa-Navarro, VonHildeverndt, A. Prieto-Cruz, J.A. MaldonadoOcampo, A.M. Umaña-Villaveces, S. Sierra y F. Forero. 2009. Caracterización de la Biodiversidad de la Selva de Mataven (Sector centro Oriental) Vichada, Colombia. Instituto Alexander Von Humboldt y Asociación de Cabildos y Autoridades Tradicionales Indigenas de la Selva de Mataven (Acatisema). Bogotá D.C. Colombia. 186p.

Mantilla-Meluk H, Jiménez-Ortega, Baker R. Phyllostomid Bats of Colombia: Annotated Checklist, Distribution and Biogeography. Special Publications Museum of Texas TechUniversity.; 56:1-37.

McNish T. 2007. Las Aves de los Llanos de la Orinoquia. MyB Riqueza Natural.

Montoya JV, Castillo MM, Sánchez L. La importancia de las inundaciones periódicas para el funcionamiento y conservación de los ecosistemas inundables de grandes río Tropicales: Estudios en la Cuenca del Orinoco. Interciencias DEC.2011;36(12):900-907. 
Mora-Fernández C, Castellanos-Castro C, Cardona-Cardozo A, Pinzón-Pérez, Vargas-Ríos JO. Págs 45-73. El medio natural: Los llanos de Casanare y área de estudio. En: León Sicard, T. (Editor). 2011. Mamíferos, Reptiles y Ecosistemas del Bloque Cubiro (Casanare): Educación Ambiental para la Conservación. Instituto de Estudios Ambientales Universidad Nacional de Colombia. AlangeEnergy Corp. Bogotá. 432pp.

Mora-Fernández C, Borrás-Ulloa M. 2011. Propuesta de un área Ramsar en las Sabanas Inundables del Departamento de Casanare-Colombia aplicando herramientas de Sistemas de Información Geográfica. Tesis de Grado. Especialización en Sistemas de Información Geográfica. Centro de Investigaciones en Información Geográfica (CIAF) del Instituto Geográfico Agustín Codazzi (IGAC). Bogotá, Colombia. 102p.

Murillo-Pacheco Jl. 2005. Evaluación de la distribución y estado actual de los registros ornitológicos de los llanos orientales de Colombia. Tesis de Pregrado. Universidad de Nariño. San Juan de Pasto. Colombia (Sin Publicar)

Nieff JJ Ideas para la interpretación ecológica del Paraná. Interciencias. 1990;15:424-441.

Novoa DF. 2002. Los Recursos Pesqueros del Eje Fluvial OrinocoApure: Presente y Futuro. Inapesca. Caracas, Venezuala. 148pp.

Ortega-Lara A. 2005. Biodiversidad ictiológica de la reserva Bojonawi, cuenca del río Orinoco, Municipio de Puerto Carreño - Vichada, Fundación Omacha, Bogotá. 34p.

Peñuela-Recio L, Fernández AP, Castro F, Ocampo . 2011a. Uso y Manejo de Forrajes Nativos en la Sabana Inundable de la Orinoquia. TheNatureConservancy, Fundación Horizonte Verde, Fundación Biodiversidad, Corporinoquia, Grupo de Investigación en Sistemas Sostenibles de producción con énfasis en palmas tropicales Universidad de los Llanos. Bogotá. 66pp.

Peñuela-Recio L, Castro LF, Ocampo-Peñuela N. 2011b. Las Reservas Naturales del Nodo Orinoquia en su rol de conservación de la Biodiversidad. Fundación Horizonte Verde y Resnatur. 104p. Colombia.

Pinzón-Pérez L, Castellanos-Castro C, Cardona-Cardozo A, MoraFernández C, Vargas-Ríos JO. Págs 99-154. Caracterización de las comunidades vegetales presentes en el Bloque Cubiro, cuenca baja del río Pauto, Casanare (Colombia). En: LEÓN SICARD, T. (Editor). 2011. Mamíferos, Reptiles y Ecosistemas del Bloque Cubiro (Casanare): Educación Ambiental para la Conservación. Instituto de Estudios Ambientales Universidad Nacional de Colombia. AlangeEnergy Corp. Bogotá. 432pp

Phillips 2007

LeRoy Poff N, Allan JD, Bain MB, Karr JR, Prestegard KL, et al. The natural flow regime. BioScience. ;47:769-784.

Postel S, Richter B. 2003. Rivers for life.Managig Water for People and Nature.Island Press, Washington, DC. EEUU: 253pp.

Rippstein G, Escobar G, Mota F. 2001. Agroecologia y biodiversidad de las sabanas de los Llanos Orientales de Colombia. Cali, Colombia: CIAT. 302pp.

Rodríguez M. 2009. "La mejor Orinoquia que podemos construir". Bogotá, pág. 138.
Romero LP, Castro F. 2012. (enEdision).Diversidad florística del municipio de Orocué (Casanare, Colombia). Facultad de Ingeniería Geográfica y Ambiental. Universidad de Ciencias Aplicadas y Ambientales (U.D.C.A). Bogotá D.C. 23p.

Restrepo-Calle S, Piña-Herrera V. 2005. Análisis de información registrada sobre riqueza, distribución, abundancia, amenazas y oportunidades para la conservación de las aves migratorias en la cuenca del río Orinoco. Informe Asociación Calidris presentado a WWF-Colombia, Cali, Colombia. 22pp.

Restrepo-Calle S, Lentino M, Naranjo LG. 2010. Aves. Pp. 291309. En: Lasso, C. A., J. S. Usma, F. Trujillo y A. Rial (Editores). 2010. Biodiversidad de la cuenca del Orinoco: bases científicas para la identificación de áreas prioritarias para la conservación y uso sostenible de la biodiversidad. Instituto de Investigación de Recursos Biológicos Alexander von Humboldt, WWF Colombia, Fundación Omacha, Fundación La Salle e Instituto de Estudios de la Orinoquia (Universidad Nacional de Colombia). Bogota, D. C., Colombia. 609 pp.

Ramia M. Tipos de Sabanas de los Llanos de Venezuela. Bol. Soc. Venez. Ci. Nat. ;27(112):264-288.

Ramírez B, Roa-López N. 2011. Educación ambiental en Casanare: retos y oportunidades para el manejo de recursos naturales en el Piedemonte y zona andina. Pag. 235-245. En: En: Usma, J.S., y F. Trujillo (Editores). 2011. Biodiversidad del Casanare: Ecosistemas Estratégicos del Departamento. Gobernación de Casanare - WWF Colombia. Bogotá D.C. 286p

Romero M, Galindo G, Otero J, Armenteras D. 2004. Ecosistemas de la cuenca del Orinoco colombiano. Instituto de Investigaciones de Recursos Biológicos Alexander von Humboldt. Bogotá. Colombia D.C. 189p.

Romero-Ruiz MH, Flantua SGA, Tansey K, Berrio JC. Landscape transformations in savannas of northern South America: Land use/cover Changes since 1987 in the Llanos Orientales of Colombia. Applied Geography. ;32(2012):766-776.

Rueda-Almonacid JV, Carr L, Mittermeier A, Rodrigez-Maecha JV, Mast RB, et al. 2007. Las torutugas t los cocodrilianos de los países andinos del trópico. Serie de guías tropicales de campo No. 6. Conservación internacional. Editorial Panamaricana, forma e Impresos. Bogotá, Colombia 538pp.

Rugeles-Lugo M, Vasques L, Bayona C. 2007. Catálogo de peces ornamentales comerciales de Arauca. Universidad Nacional de Colombia - sede Orinoquia. 51p.

Sánchez R, Castro P, Galvis G. Similaridad de la composición íctica entre dos ambientes del río Yucao, sistema del río Meta, Colombia. Revista Académica Colombiana de Ciencias. ;23(Suplemento especial):567-574.

Sarmiento G. 1984. Ecology of Neotropical Savannas. Harvard university Press. Boston. USA. Xii + 235p.

Sarmiento G. 1994. Sabanas Naturales: génesis y ecología. En: Sabanas Naturales de Colombia (p. 17-55). Cali. Banco de Occidente.

Sastre H, Huertas H. 2006. Fomento de Rescate del ganado criollo Casanare. Yopal. Pp 1-19.

Schöngart J, Junk WJ. Forecasting the flood-pulse in Central Amazonia by ENSO-indices. J. Hydrol. ;335:124-132. 
Stanford JA, Lorang MS, Hauer FR. The shifting habitat mosaic of river ecosystems.Verhand. Int. Verein. Theor. Angew. Limnol.; 29:123-136.

Suárez-Castro FA, Sánchez-Palomino P. Págs 277-300. Diversidad de Mamíferos presentes en el Bloque Cubiro y amenazas para su conservación. En: León Sicard, T. (Editor). 2011. Mamíferos, Reptiles y Ecosistemas del Bloque Cubiro (Casanare): Educación Ambiental para la Conservación. Instituto de Estudios Ambientales Universidad Nacional de Colombia. AlangeEnergy Corp. Bogotá. 432pp.

Trujillo F, Garavito-Fonseca J, Gutiérrez K, Rodríguez-Maldonado , Combariza RL,et al. 2010. Anfibios y Reptiles. 181-205p. En: Usma, J.S., y F. Trujillo (Editores). 2010. Biodiversidad del Casanare: Ecosistemas Estratégicos del Departamento. Gobernación de Casanare - WWF Colombia. Bogotá D.C. 286p

Tocker K, Stanford JA. Riverine flood plains: present state and future trends. Env. Cons. ;29:208-330.

Urbano-Bonilla A, Zamudio J, Maldonado J, Ocampo A, CortésMillán G. Estudio preliminar de la laguna el Tinije Aguazul (Casanare). Colombia: Diversidad y Aspectos ecológicos. Actualidades Biológicas. ;31(Suplemento 1):217p.

Urbano-Bonilla A, Zamudio J, Maldonado J, Ocampo A, Bogotá-Grégory JD, et al. Peces del Pie de Monte de Casanare, Colombia. Biota Colombiana. ;10:149-162.
Usma JS, Trujillo F. (Editores). 2011. Biodiversidad del Casanare: Ecosistemas Estratégicos del Departamento. Gobernación de Casanare - WWF Colombia. Bogotá D.C. 286p.

Villa-Navarro F, Urbano-Bonilla A, Ortega-Lara A, Taphorn D, UsmaOviedo JS. 2011. Peces del Casanare. Pp. 120-136. En: Usma, J.S., y F. Trujillo (Editores). 2011. Biodiversidad del Casanare: Ecosistemas Estratégicos del Departamento. Gobernación de Casanare - WWF Colombia. Bogotá D.C. 286p.

Viña G, Santos S, Ramírez A, Botero I, Otíz V, et al. 1997. Programa de monitoreo biológico campos Cusiana.Cupiagua y Bloque Piedemonte: Resultados de los estudios de Fauna y Flora acuática. BP. Exploration.Bogotá.

Welcomme RL. 1979. Fisheries ecology of flood-plain rivers. Longman, London, RU.

Winemiller, KO. 2004. Floodplain river food webs: Generalizations and implications for fisheries management. En Welcomme RL, Petr T (Eds.) Proc. 2nd Int. Symp. Management of Large Rivers for Fisheries.Volume II. RAP Publication 2004/17. FAO Regional Office for Asia and the Pacific. Bangkok, Thailand. 285-309pp.

Zamudio JA, Ortega LF, Castillo LF. Aves del Casanare. 2010. Pp. 168-179. En: Usma, J.S., y F. (Editores). 2010. Biodiversidad del Casanare: Ecosistemas Estratégicos del Departamento. Gobernación de Casanare - WWF Colombia. Bogotá D.C. 286p. 